CERN-PH-TH/2005-115

\title{
Gradient expansion(s) and dark energy
}

\author{
Massimo Giovannini ${ }^{1}$ \\ Centro "Enrico Fermi", Compendio del Viminale, Via Panisperna 89/A, 00184 Rome, Italy \\ Department of Physics, Theory Division, CERN, 1211 Geneva 23, Switzerland
}

\begin{abstract}
Motivated by recent claims stating that the acceleration of the present Universe is due to fluctuations with wavelength larger than the Hubble radius, we present a general analysis of various perturbative solutions of fully inhomogeneous Einstein equations supplemented by a perfect fluid. The equivalence of formally different gradient expansions is demonstrated. If the barotropic index vanishes, the deceleration parameter is always positive semi-definite.
\end{abstract}

\footnotetext{
${ }^{1}$ e-mail address: massimo.giovannini@cern.ch
} 


\section{Introduction}

Type Ia supernovae seem to suggest that the present Universe is experiencing a phase of accelerated expansion $[1,2]$ (see also [3, 4] for earlier results reporting the first evidences of this phenomenon). The experimental results are also consistent with the determination of the cosmological parameters coming from CMB physics [5]. These results seem to imply that up to $70 \%$ of the present energetic content of the Universe is "dark". If the dark energy component is parametrized with a perfect fluid characterized by a barotropic index $w$, the experimental determinations reported in Refs. $[1,2]$ suggest that for a flat Universe with $\Omega_{\text {tot }}=1, \Omega_{\text {matter }}=0.29 \pm_{0.03}^{0.05}$ and $w=-1.02 \pm_{0.19}^{0.13}$.

The simplest model of dark energy is, in some sense, a time-independent cosmological constant whose associated $w$ is exactly -1 . Fluid models of dark energy may lead to barotropic indices in the range $-1 \leq w \leq-1 / 3$. It is equally plausible that the dark-energy component is described by a scalar degree of freedom (i.e. the quintessence field) whose potential becomes dominant around the present time [6] (see also [7]). Such a degree of freedom may also be a pseudo-scalar [8]. Quintessence models of dark energy imply generically that $w>-1$. A rather economical class of models is the one where the quintessence field (dominant today) and the inflaton field (dominant in the far past history of the Universe) are identified in a single (scalar) degree of freedom. These are the so-called quintessential inflationary models [9] whose salient features may lead necessarily to a stochastic background of gravitational radiation for typical frequencies larger than $0.1 \mathrm{KHz}[10,11,12]$. Useful surveys on the status of fluid and quintessential models of dark energy can be found, respectively, in Refs. [13] and [14].

Experimental data cannot rule out values $w<-1$ : this occurrence stimulated the investigation of fluid models of dark energy where the cosmological energy density is future increasing, rather than future decreasing, as in the case $w>-1$. Provided $w<-1$ all the time in the future, a "big rip" can be expected [15], i.e. an infinite expansion of the Universe in a finite amount of time $[16,17]$. Along this perspective, recently Barrow has discussed the interesting case of "sudden" singularities that may arise in expanding FRW Universes even if the dominant energy condition is not violated [19, 20]. The possibility of anisotropic sudden singularities has been also discussed recently by Barrow and Tsagas [21]. We will briefly comment about the possible relevance of the considerations developed here in the context of the physics of sudden singularity.

The aim of the present paper is to understand wether an inhomogeneous Universe may sustain a phase of accelerated expansion even if the barotropic index of the sources is positive semi-definite, i.e. $w \geq 0$. There are various reasons to discuss this problem. It is, in fact, plausible that inhomogeneities (with typical wavelengths larger than the Hubble radius) may be generated in the early Universe provided the duration of the inflationary phase exceeds, say, 65 e-folds. After equality and before decoupling, (when inflationary perturbations are imprinted, via the Sachs-Wolfe effect, on the temperature anisotropies) 
the metric fluctuations are perturbative. Still, one could wonder if higher-order effects in the amplitude of the fluctuations may change this perspective and make the present amplitude of inflationary generated fluctuations so large to affect, for instance, the deceleration parameter.

There may be a way out in this technical impasse. The idea is to forget about homogeneous backgrounds (supplemented by tiny inhomogeneous fluctuations) and to consider, instead, some type of gradient expansion. This theoretical tool has a long history that can be traced back to the seminal contributions of Lisfhitz, Belinskii, Khalatnikov and collaborators (see $[22,23]$ and references therein). This approach allows to compute the phenomenologically relevant quantities to a given order in the spatial gradients. There have been recently claims [24] suggesting that indeed a matter-dominated Universe (without any vorticity) may be accelerating solely thanks to the presence of super-Hubble inhomogeneities 2 . In $[25,26,27,28,29,30,31]$ various independent criticisms of this proposal have been presented. In [25] it was argued that fluctuations with typical wavelength larger than the Hubble radius can certainly affect the spatial curvature but can never accelerate the Universe. Similar conclusion, through a different chain of arguments, has been derived in [26]. In [27] demonstrated that super-horizon fluctuations do not produce an accelerated expansion. Their approach has been to take an exact solution of Einstein equations ${ }^{3}$ (describing an underdense region). This strategy allowed to obtain a specific (computable) model of super-horizon fluctuations falling in the class discussed in [24]. The example showed that no acceleration takes place in agreement with general theorems implying that the Universe cannot accelerate unless the strong energy condition is violated. In [27] an explicit analysis of the variance of the deceleration parameter has been also performed in the case of singlefield inflationary models. In [29] the perspective has been to analyse a form of averaged Einstein equations by assuming the correctness of some of the conclusions of [24]. The conclusion of this study has been that the model of [24] is likely to be ruled out by present observations.

The analysis of Ref. [30] dealt also with the effect of small-scale fluctuations (i.e. with wavelengths smaller than the Hubble radius). The conclusion of the analysis has been that sub-horizon perturbations are not a viable candidate for the explanation of the present acceleration of the Universe.

In [31], an approach for the analysis of fully inhomogeneous Einstein equations has been proposed and applied to the case of Ref. [24]. The calculation of [24] has been then repeated and it has been discovered that, within the accuracy of the solution, the late-time behaviour of the spatial gradients in a matter dominated Universe can never make the deceleration parameter negative. Furthermore, by enforcing the validity of the perturbative expansion it

\footnotetext{
${ }^{2}$ To stress the fact that the Universe is accelerated thanks to super-horizon fluctuations, the authors of [24] called their proposal Super Hubble Cold Dark Matter (SHCDM) to be contrasted with the usual $\Lambda$ CDM.

${ }^{3}$ Recently, a similar perspective has been adopted in the analysis of a Lemaitre-Tolman-Bondi Universe by Alnes, Amarzguioui and Gron [28] (see also [18] ).
} 
has been shown that, at later times the deceleration parameter vanishes, i.e. the expected result is the Universe is dominated by gradients.

The purpose of the present paper is to extend and generalize the analysis presented in [31] along different lines. The first generalization consist in discussing the gradient expansion without the restriction of a dust-dominated Universe but for a generic barotropic index. Furthermore, in [31] the analysis has been performed by only keeping only one scalar degree of freedom of the fully inhomogeneous metric. Here, as announced [31] all the degrees of freedom of the inhomogeneous metric will be analyzed simultaneously. The related aim of the present analysis is to stress the mutual connections of apparently diverse gradient expansions.

The plan of the present paper is the following. In Section 2 the fully inhomogeneous Einstein equations will be introduced in terms of the extrinsic and intrinsic curvature tensors. Section 3 deals with the interesting case of anti-newtonian solutions that could be an appropriate seed solution for a gradient expansion aiming at the description of super-horizon fluctuations. In Section 4 the gradient expansion will be derived. Solutions accurate up to two gradients will be presented. In Section 5 the gradient expansion will be applied to a situation where the parametrization of the seed metric is apparently different. Section 6 contains some more phenomenological considerations dealing with the late-time behaviour of a Universe dominated by super-Hubble fluctuations. Finally, Section 7 contains some concluding remarks.

\section{Inhomogeneous Einstein equations}

Consider a four-dimensional line element of the form

$$
d s^{2}=d t^{2}-\gamma_{i j}(t, \vec{x}) d x^{i} d x^{j}
$$

the symmetric rank-two tensor $\gamma_{i j}$ in three dimensions contains 6 independent degrees of freedom. The components of the extrinsic curvature are

$$
K_{i j}=-\frac{1}{2} \frac{\partial}{\partial t} \gamma_{i j}, \quad K_{i}^{j}=-\frac{1}{2} \gamma^{i k} \frac{\partial}{\partial t} \gamma_{k j}
$$

In the following, the compact notation $\operatorname{Tr} K^{2}=K_{i}^{j} K_{j}^{i}$ will be used. The three-dimensional Ricci tensor becomes instead

$$
r_{i j}=\partial_{m} \Gamma_{i j}^{m}-\partial_{j} \Gamma_{m i}^{m}+\Gamma_{i j}^{m} \Gamma_{m \ell}^{\ell}-\Gamma_{j m}^{\ell} \Gamma_{i \ell}^{m},
$$

where

$$
\Gamma_{i j}^{m}=\frac{1}{2} \gamma^{m \ell}\left(-\partial_{\ell} \gamma_{i j}+\partial_{j} \gamma_{\ell i}+\partial_{i} \gamma_{j \ell}\right)
$$

Equations (2.2) and (2.3) allow to write the Einstein equations in a fully inhomogeneous form. More specifically, assuming that the energy-momentum tensor is a perfect relativistic 
fluid

$$
T_{\mu}^{\nu}=(p+\rho) u_{\mu} u^{\nu}-p \delta_{\mu}^{\nu},
$$

the Hamiltonian and momentum constraints are, respectively,

$$
\begin{aligned}
& K^{2}-\operatorname{Tr} K^{2}+r=16 \pi G\left[(p+\rho) u_{0} u^{0}-p\right], \\
& \nabla_{i} K-\nabla_{k} K_{i}^{k}=8 \pi G u_{i} u^{0}(p+\rho) .
\end{aligned}
$$

The $(i j)$ components of Einstein equations lead instead to

$$
\begin{aligned}
& \left(\dot{K}_{i}^{j}-K K_{i}^{j}-\dot{K} \delta_{i}^{j}\right)+\frac{1}{2} \delta_{i}^{j}\left(K^{2}+\operatorname{Tr} K^{2}\right)-\left(r_{i}^{j}-\frac{1}{2} r \delta_{i}^{j}\right) \\
& =-8 \pi G\left[(p+\rho) u_{i} u^{j}+p \delta_{i}^{j}\right]
\end{aligned}
$$

where the overdot denotes a partial derivation with respect to $t$ while $\nabla_{i}$ denotes the covariant derivative defined in terms of $\gamma_{i j}$ and of Eq. (2.4). A trivial remark is that, in Eqs. (2.6), (2.7) and (2.8), the indices are raised and lowered using directly $\gamma_{i j}(t, \vec{x})$.

By combining the previous set of equations the following relation can be easily deduced

$$
q \operatorname{Tr} K^{2}=8 \pi G\left[(p+\rho) u_{0} u^{0}+\frac{p-\rho}{2}\right]
$$

where

$$
q(\vec{x}, t)=-1+\frac{\dot{K}}{\operatorname{Tr} K^{2}}
$$

is the inhomogeneous generalization of the deceleration parameter. In fact, in the homogeneous and isotropic limit, $\gamma_{i j}=a^{2}(t) \delta_{i j}, K_{i}^{j}=-H \delta_{i}^{j}$ and, as expected, $q(t) \rightarrow-\ddot{a} a / \dot{a}^{2}$. Recalling the definition of $\operatorname{Tr} K^{2}$ (given after Eq. (2.2)) it is rather easy to show that

$$
\operatorname{Tr} K^{2} \geq \frac{K^{2}}{3} \geq 0
$$

where the sign of equality (in the first relation) is reached, again, in the isotropic limit.

Since $\gamma^{i j}$ is always positive semi-definite, it is also clear that

$$
u_{0} u^{0}=1+\gamma^{i j} u_{i} u_{j} \geq 1
$$

that follows from the condition $g^{\mu \nu} u_{\mu} u_{\nu}=1$. From Eq. (2.9) it follows that $q(t, \vec{x})$ is always positive semi-definite if $(\rho+3 p) \geq 0$. As correctly pointed out in [27] and [29] that this statement assumes the absence of rotational fluctuations. This is rather plausible since super-horizon vector modes are very unlikely to be generated either during inflation or during a phase where the Universe expands. Concerning the possible rôle of vector modes in conventional and unconventional inflationary models see, for instance, [32, 33, 34, 35]. 
Equations (2.6), (2.8) and (2.7) must be supplemented by the explicit form of the covariant conservation equations:

$$
\begin{aligned}
& \frac{1}{\sqrt{\gamma}} \frac{\partial}{\partial t}\left[\sqrt{\gamma}(p+\rho) u^{0} u^{i}\right]-\frac{1}{\sqrt{\gamma}} \partial_{k}\left\{\sqrt{\gamma}\left[(p+\rho) u^{k} u^{i}+p \gamma^{k i}\right]\right\}-2 K_{\ell}^{i} u^{0} u^{\ell}(p+\rho) \\
& -\Gamma_{k \ell}^{i}\left[(p+\rho) u^{k} u^{\ell}+p \gamma^{k \ell}\right]=0, \\
& \frac{1}{\sqrt{\gamma}} \frac{\partial}{\partial t}\left\{\sqrt{\gamma}\left[(p+\rho) u_{0} u^{0}-p\right]\right\}-\frac{1}{\sqrt{\gamma}} \partial_{i}\left\{\sqrt{\gamma}(p+\rho) u_{0} u^{i}\right\} \\
& -K_{k}^{\ell}\left[(p+\rho) u^{k} u_{\ell}+p \delta_{\ell}^{k}\right]=0,
\end{aligned}
$$

where $\gamma=\operatorname{det}\left(\gamma_{i j}\right)$.

It is useful to recall, from the Bianchi identities, that the intrinsic curvature tensor and its trace satisfy the following identity

$$
\nabla_{j} r_{i}^{j}=\frac{1}{2} \nabla_{i} r
$$

Note, finally, that combining Eq. (2.6) with the trace of Eq. (2.8) the following equation is obtained:

$$
\operatorname{Tr} K^{2}+K^{2}+r-2 \dot{K}=8 \pi G(\rho-3 p) .
$$

Equation (2.16) allows to re-write Eqs. (2.6), (2.8) and (2.7) as

$$
\begin{aligned}
& \dot{K}-\operatorname{Tr} K^{2}=8 \pi G\left[(p+\rho) u_{0} u^{0}+\frac{p-\rho}{2}\right], \\
& \frac{1}{\sqrt{\gamma}} \frac{\partial}{\partial t}\left(\sqrt{\gamma} K_{i}^{j}\right)-r_{i}^{j}=8 \pi G\left[-(p+\rho) u_{i} u^{j}+\frac{p-\rho}{2} \delta_{i}^{j}\right], \\
& \nabla_{i} K-\nabla_{k} K_{i}^{k}=8 \pi G(p+\rho) u_{i} u^{0} .
\end{aligned}
$$

\section{Anti-newtonian solutions}

Consider the situation where the scalar curvarure $r$ and the velocities are all much smaller than $K^{2}$ and $\dot{K}$. This situation describes the occurrence of a "anti-newtonian" regime and has been previously investigated by Tomita $[36,37]$ as well as by Deruelle and collaborators $[38,39,40]$ interested in various aspects of this approximation.

If $K^{2}$ and $\dot{K}$ are both leading in comparison with the curvature and velocity contributions, Eqs. (2.17), (2.18) and (2.13) can be written, as

$$
\begin{aligned}
& \dot{K}-\frac{K^{2}}{3}-\operatorname{Tr} Q^{2}=4 \pi G(3 p+\rho), \\
& \frac{1}{\sqrt{\gamma}} \frac{\partial}{\partial t}(\sqrt{\gamma} K)=12 \pi G(p-\rho), \\
& \frac{1}{\sqrt{\gamma}} \frac{\partial}{\partial t}\left(\sqrt{\gamma} Q_{i}^{j}\right)=0,
\end{aligned}
$$


where we defined

$$
K_{i}^{j}=\frac{K}{3} \delta_{i}^{j}+Q_{i}^{j},
$$

with $Q=Q_{i}^{i}=0$. From Eq. (2.14), covariant conservation of the energy-momentum tensor implies

$$
\dot{\rho}+\frac{\dot{\gamma}}{2 \gamma}(p+\rho)=0
$$

From Eq. (3.3) and (3.5) it follows

$$
\begin{aligned}
& \rho=\rho_{0}(\vec{x}) \gamma^{-\frac{w+1}{2}}, \\
& Q_{i}^{j}=\frac{\lambda_{i}^{j}}{\sqrt{\gamma}}
\end{aligned}
$$

where $\lambda_{i}^{i}=0$ and $\lambda_{i}^{j}$ only depends on space (and not on time); note that we used the fact that $p=w \rho$.

Inserting then Eq. (3.2) into Eq. (3.1) to eliminate the energy density we obtain:

$$
\dot{K}-\frac{w+1}{2} K^{2}+\frac{3}{4}(w-1) \frac{\operatorname{Tr} \lambda^{2}}{\gamma}=0,
$$

where, as usual, $\operatorname{Tr} \lambda^{2}=\lambda_{i}^{j} \lambda_{j}^{i}$. A new variable can now be defined, namely,

$$
M=\gamma^{\frac{w+1}{4}}
$$

recalling that $\dot{\gamma}=-2 \gamma K$, Eq. (3.8) becomes, in terms of $M$ :

$$
\ddot{M}=\frac{3}{8}\left(w^{2}-1\right) \operatorname{Tr} \lambda^{2} M^{\frac{w-3}{w+1}} .
$$

Integrating once with respect to the cosmic time, the following relation can be obtained:

$$
\dot{M}^{2}=\frac{3}{8}(w+1)^{2} \operatorname{Tr} \lambda^{2} M^{\frac{2(w-1)}{w+1}}+N(\vec{x}) .
$$

Equation (3.11) can be solved (either analytically or numerically) for a given value of the barotropic index. In particular, in the case $w=0$, recalling Eq. (3.9) we can write:

$$
\sqrt{\gamma}=N(\vec{x})\left[t-t_{1}(\vec{x})\right]^{2}-\frac{3}{8} \frac{\operatorname{Tr} \lambda^{2}}{N(\vec{x})}
$$

where a further integration constant appear. Either $N(\vec{x})$ or $t_{1}(\vec{x})$ can be fixed by exploiting the remaining gauge freedom of the synchronous system. Note that the momentum constraint of Eq. (2.19) implies

$$
u^{0} u_{i}=\frac{1}{12 \pi G \rho(w+1)}\left[\partial_{i} K-\frac{3}{2 \sqrt{\gamma}}\left(\Gamma_{k \ell}^{k} \lambda_{i}^{\ell}-\Gamma_{i m}^{k} \lambda_{k}^{m}\right)\right]
$$

that is of higher order in the gradients, as expected. 
The deceleration parameter defined in Eq. (2.10) can be written, using Eqs. (2.10) and (3.7) as

$$
q(\vec{x}, t)=\frac{1}{4}\left(\frac{2 \gamma K^{2}-3 \operatorname{Tr} \lambda^{2}}{\gamma K^{2}+3 \operatorname{Tr} \lambda^{2}}\right)
$$

i.e., using Eq. (3.12)

$$
q(\vec{x}, t)=\frac{1}{4}\left(\frac{8 N(\vec{x})^{2}\left[t-t_{1}(\vec{x})\right]^{2}-3 \operatorname{Tr} \lambda^{2}}{4 N(\vec{x})^{2}\left[t-t_{1}(\vec{x})\right]^{2}+3 \operatorname{Tr} \lambda^{2}}\right),
$$

whose large time limit is $1 / 2$. Note that by looking superficially at Eq. (3.15) one would be tempted to conclude that $q$ might be negative. This is not correct since the numerator in of the expression in Eq. (3.15) is exactly proportional to $\sqrt{\gamma} \geq 0$.

Now one could choose, as seed metric, the solution found with this method. Then compute the spatial curvature and obtain the following order in the gradient expansion and so on $[38,40]$. However, the solution of Eq. (3.11) cannot be inverted analytically for a generic barotropic index $w$.

\section{Quasi-isotropic solution and gradient expansion}

The solutions of Eqs. (2.6)-(2.7) can be classified, according to their degree of isotropy, in quasi-isotropic solutions and fully anisotropic solutions. For instance, close to the initial (big-bang) singularity the solution of Eqs. (2.6)-(2.7) is, in general, fully anisotropic. In the opposite limit, i.e. far from the initial singularity, the possibility of quasi-isotropic solutions becomes more relevant. Quasi-isotropic solutions exist, indeed, only in the presence of matter $[41,42]$ (see also $[43,44,45])$.

Let us now look for solutions of the previous system of equations in the form of a gradient expansion. In other words we shall be considering $\gamma_{i j}$ written in the form

$$
\gamma_{i k}=a^{2}(t)\left[\alpha_{i k}(\vec{x})+\beta_{i k}(t, \vec{x})\right], \quad \gamma^{k j}=\frac{1}{a^{2}(t)}\left[\alpha^{k j}-\beta^{k j}(t, \vec{x})\right],
$$

where $\beta(\vec{x}, t)$ is considered to be the first-order correction in the spatial gradient expansion. Note that from Eq. (4.1) $\gamma_{i k} \gamma^{k j}=\delta_{i}^{j}+\mathcal{O}\left(\beta^{2}\right)$. The logic is now very simple since Einstein equations will determine the specific form of $\beta_{i j}$ once the specific form of $\alpha_{i j}$ is known.

Using Eq. (4.1) into Eqs. (2.2) we obtain

$$
K_{i}^{j}=-\left(H \delta_{i}^{j}+\frac{\dot{\beta}_{i}^{j}}{2}\right), \quad K=-\left(3 H+\frac{1}{2} \dot{\beta}\right), \quad \operatorname{Tr} K^{2}=3 H^{2}+H \dot{\beta}
$$

where, with obvious notations, $H=\dot{a} / a$.

From Eq. (2.7) it also follows

$$
\nabla_{k} \dot{\beta}_{i}^{k}-\nabla_{i} \dot{\beta}=16 \pi G u_{i} u^{0}(p+\rho) .
$$


The explicit form of the momentum constraint suggests to look for the solution in a separable form, namely, $\beta_{i}^{j}(t, \vec{x})=g(t) \mathcal{B}_{i}^{j}(\vec{x})$. Thus Eq. (4.3) becomes

$$
\dot{g}\left(\nabla_{k} \mathcal{B}_{i}^{k}-\nabla_{i} \mathcal{B}\right)=16 \pi G u_{i} u^{0}(p+\rho) .
$$

Using this parametrization and solving the constraint for $u_{i}$, Einstein equations to second order in the gradient expansion reduce then to the following equation:

$$
(\ddot{g}+3 H \dot{g}) \mathcal{B}_{i}^{j}+H \dot{g} \mathcal{B} \delta_{i}^{j}+\frac{2 \mathcal{P}_{i}^{j}}{a^{2}}=\frac{w-1}{3 w+1}(\ddot{g}+2 H \dot{g}) B \delta_{i}^{j} .
$$

In Eq. (4.5) the spatial curvature tensor has been parametrized as

$$
r_{i}^{j}=\frac{\mathcal{P}_{i}^{j}}{a^{2}}
$$

Recalling that

$$
H=H_{0} a^{-\frac{3(w+1)}{2}}, \quad \dot{H}=-\frac{3(w+1)}{2} H^{2},
$$

the solution for Eq. (4.5) can be written as

$$
\begin{aligned}
& \mathcal{B}_{i}^{j}=-\frac{4}{H_{0}^{2}(3 w+1)(3 w+5)}\left(\mathcal{P}_{i}^{j}-\frac{5+6 w-3 w^{2}}{4(9 w+5)} \mathcal{P} \delta_{i}^{j}\right), \\
& \mathcal{B}=-\frac{\mathcal{P}}{H_{0}^{2}(9 w+5)}
\end{aligned}
$$

with $g(t)$ simply given by

$$
g(t)=a^{3 w+1}
$$

Note that, in Eq. (4.8), $H_{0}=2 /\left[3(w+1) t_{0}\right]$. Eqs. (4.8) agree with the expression obtained in the case $w=1 / 3$ in Ref. [22] (where the notations are such that $t_{0}=1$ ). Equation (4.8) can be also inverted, i.e. $\mathcal{P}_{i}^{j}$ can be easily expressed in terms of $\mathcal{B}_{i}^{j}$ and $\mathcal{B}$ :

$$
\mathcal{P}_{i}^{j}=-\frac{H_{0}^{2}}{4}\left[\mathcal{B} \delta_{i}^{j}\left(6 w+5-3 w^{2}\right)+\mathcal{B}_{i}^{j}(3 w+5)(3 w+1)\right]
$$

Using Eq. (2.15) the peculiar velocity field and the energy density can also be written as

$$
\begin{aligned}
& u^{0} u_{i}=-\frac{3}{8 \pi G \rho}\left(\frac{w}{3 w+5}\right) a^{3 w+1} H \partial_{i} \mathcal{B}(\vec{x}), \\
& \rho=\frac{3 H_{0}^{2}}{8 \pi G}\left[a^{-3(w+1)}-\frac{w+1}{2} \mathcal{B}(\vec{x}) a^{-2}\right] .
\end{aligned}
$$

Let us therefore rewrite the solution in terms of $\gamma_{i j}$, i.e.

$$
\gamma_{i j}=a^{2}(t)\left[\alpha_{i j}(\vec{x})+\beta_{i j}(\vec{x}, t)\right]=a^{2}(t)\left[\alpha_{i j}(\vec{x})+a^{3 w+1} \mathcal{B}_{i j}(\vec{x})\right] .
$$

Concerning this solution a few comments are in order: 
- if $w>-1 / 3, \beta_{i j}$ becomes large as $a \rightarrow \infty$ (note that if $w=-1 / 3, a^{3 w+1}$ is constant);

- if $w<-1 / 3, \beta_{i j}$ vanishes as $a \rightarrow \infty$;

- if $w<-1, \beta_{i j}$ not only the gradients become sub-leading but the energy density also increases as $a \rightarrow \infty$.

- to the following order in the perturbative expansion the time-dependence is easy to show: $\gamma_{i j} \simeq a^{2}(t)\left[\alpha_{i j}+a^{3 w+1} \mathcal{B}_{i j}+a^{2(3 w+1)} \mathcal{E}_{i j}\right]$ and so on for even higher order terms; clearly the calculation of the curvature tensors will now be just a bit more cumbersome.

The first two comments are rather elementary. The first comment simply expresses the fact that if $w>-1 / 3$ the gradients decay close to the initial big-bang singularity (but not far from it!). The second comment simply justifies why during a phase dominated by an effective cosmological constant (or by a fluid violating the strong energy condition) the gradients are washed out. The third comment may have some interesting implications for the study of big rip singularities or for the more general case of sudden $[19,20,21]$ singularities. It would be for instance interesting to investigate if, in general, sudden singularities will exhibit or not some type of BKL oscillations that are known to be present in the case of the initial big-bang singularity.

\section{Equivalent forms of gradient expansion}

Consider now the following parametrization of the perturbed metric:

$$
\gamma_{i j}=e^{-2 \Psi(t, \vec{x})} a^{2}(t)\left[\delta_{i j}+\mu_{i j}(\vec{x}, t)\right],
$$

where $\mu_{i}^{i}=0$. This parametrization has been used in [24] and in [31]. Equation (5.1) may seem, superficially, different from the one of Eq. (4.1) since, on one hand, the analog of $\alpha_{i j}$ has a very specific tensor structure coinciding with the three-dimensional Kroeneker symbol; on the other hand $\Psi$ is allowed to depend both on space and time.

Equation (5.1)

$$
K_{i}^{j}=(\dot{\Psi}-H) \delta_{i}^{j}-\frac{\dot{\mu}_{i}^{j}}{2} .
$$

In this case, the Hamiltonian constraint of Eq. (2.6) leads to

$$
6(\dot{\Psi}-H)^{2}+\frac{e^{2 \Psi}}{a^{2}}\left[4 \nabla^{2} \Psi-2(\nabla \Psi)^{2}\right]=16 \pi G\left[\rho+(p+\rho) \frac{e^{2 \Psi}}{a^{2}}\left(u^{2}-\tilde{u}^{2}\right)\right],
$$

where $u^{2}=u_{i} u_{j} \delta^{i j}$ and $\tilde{u}^{2}=u_{i} u_{j} \mu^{i j}$. From the $(i j)$ components of Einstein equations we get, after linear combination,

$$
3(\ddot{\Psi}-\dot{H})-3(\dot{\Psi}-H)^{2}=4 \pi G(\rho+3 p)+8 \pi G(p+\rho) \frac{e^{2 \Psi}}{a^{2}}\left(u^{2}-\tilde{u}^{2}\right),
$$




$$
\begin{aligned}
& \ddot{\mu}_{i}^{j}+3 H \dot{\mu}_{i}^{j}=-2 \frac{e^{2 \Psi}}{a^{2}}\left[\partial_{i} \partial^{j} \Psi-\frac{1}{3} \nabla^{2} \Psi \delta_{i}^{j}+\partial_{i} \Psi \partial^{j} \Psi-\frac{1}{3}(\nabla \Psi)^{2} \delta_{i}^{j}\right] \\
& +16 \pi G(p+\rho) \frac{e^{2 \Psi}}{a^{2}}\left[\left(u_{i} u^{j}-\tilde{u}_{i} \tilde{u}^{j}\right)-\frac{u^{2}-\tilde{u}^{2}}{3} \delta_{i}^{j}\right],
\end{aligned}
$$

where $\nabla^{2} \Psi=\delta^{i j} \partial_{i} \partial_{j} \Psi$ and where $(\nabla \Psi)^{2}=\delta^{i j} \partial_{i} \Psi \partial_{j} \Psi$. Finally, the momentum constraint, to first-order in the gradient expansion, is

$$
\partial_{i} \dot{\Psi}+\frac{1}{4} \partial_{k} \dot{\mu}_{i}^{k}=4 \pi G(p+\rho) u_{i} u^{0} .
$$

A more tractable form of the system can be obtained by eliminating the energy density; hence the following pair of equations emerges:

$$
\begin{aligned}
& -\ddot{\Psi}+\frac{3(w+1)}{2} \dot{\Psi}^{2}-3 H(w+1) \dot{\Psi}+\frac{3 w+1}{3 a^{2}} e^{2 \Psi}\left[\nabla^{2} \Psi-\frac{1}{2}(\nabla \Psi)^{2}\right]=0, \\
& \ddot{\mu}_{i}^{j}+3 H \dot{\mu}_{i}^{j}=-\frac{2}{a^{2}} e^{2 \Psi}\left[\partial_{i} \partial^{j} \Psi-\frac{1}{3}\left(\nabla^{2} \Psi\right) \delta_{i}^{j}+\partial_{i} \Psi \partial^{j} \Psi-\frac{1}{3}(\nabla \Psi)^{2} \delta_{i}^{j}\right] .
\end{aligned}
$$

The solution of Eqs. (5.6) and (5.7) can be written as:

$$
\begin{aligned}
& \Psi=f+\frac{j_{1}(w)}{H_{0}^{2}} a^{3 w+1} e^{2 f}\left[\nabla^{2} f-\frac{1}{2}(\nabla f)^{2}\right], \\
& \mu_{i}^{j}=-\frac{j_{2}(w)}{H_{0}^{2}} a^{3 w+1} e^{2 f}\left[\partial_{i} \partial^{j} f-\frac{1}{3}\left(\nabla^{2} f\right) \delta_{i}^{j}+\partial_{i} f \partial^{j} f-\frac{1}{3}(\nabla f)^{2} \delta_{i}^{j}\right] .
\end{aligned}
$$

where

$$
j_{1}(w)=\frac{2}{3(9 w+5)}, \quad j_{2}(w)=\frac{4}{(3 w+1)(3 w+5)}
$$

and where $f(\vec{x})$ is an arbitrary function depending upon space but not upon time. In this function is encoded the information on the specific kind of super-horizon fluctuations. The velocity fields have been consistently neglected since they are of higher order in the gradient expansion. This aspect can be appreciated by inserting the solution (5.8) into Eq. (5.5): $\partial_{i} \dot{\Psi}$ only receives contribution from the second term in Eq. (5.8) (i.e. the one proportional to $j_{1}(w)$ ). Thus $u_{i}$ will contain three gradients and will be negligible at this order. Notice, however, that the contribution of the velocity field must be necessarily taken into account when going to orders higher than the second in the gradient expansion.

Since Eq. (5.8) and (5.9) are derived under the approximation that terms with more than two gradients are neglected, the terms $\dot{\Psi}^{2}$ has to be negligible with respect to $\ddot{\Psi}$, i.e.

$$
2|\ddot{\Psi}| \gg 3(w+1)|\dot{\Psi}|^{2}
$$

This aspect can be appreciated by computing $\dot{\Psi}^{2}$ from Eq. (5.8): the only contribution to $\dot{\Psi}^{2}$ comes from the term proportional to $j_{1}(w)$ in Eq. (5.8) and this has four spatial gradients. A relevant consequence of this basic observation is that when computing $\operatorname{Tr} K^{2}$ from Eq. (5.2) the correct result, within the approximations made so far, is

$$
\operatorname{TrK}^{2}=H^{2}-2 H \dot{\Psi} \geq 0
$$


and not $\operatorname{Tr} K^{2}=H^{2}-2 H \dot{\Psi}+\dot{\Psi}^{2}$. Notice that the inequality appearing in Eq. (5.12) is a direct consequence of the inequalities reported in Eq. (2.11): $\operatorname{Tr} K^{2}$ is always (i.e. at any order) positive semi-definite. This simple observation has a simple consequence, i.e. that

$$
\dot{\Psi} \leq \frac{H}{2} .
$$

For future convenience, let us rewrite the solution of Eq. (5.8) as

$$
\Psi(\vec{x}, t)=f(\vec{x})+a^{3 w+1} \bar{\Psi}_{0}(\vec{x})
$$

where $\bar{\Psi}_{0}$ can be read-off from Eq. (5.8)

$$
\bar{\Psi}_{0}(\vec{x})=\frac{j_{1}(w)}{H_{0}^{2}} e^{2 f}\left[\nabla^{2} f-\frac{1}{2}(\nabla f)^{2}\right] .
$$

From Eq. (5.14), by taking the first time derivative, it follows easily that $\dot{\Psi}=(3 w+$ 1) $a^{3 w+1} H \bar{\Psi}_{0}$, and this implies: inserting the obtained expression for $\dot{\Psi}$ into Eq. (5.13), the inequality puts a condition on $\bar{\Psi}_{0}$, i.e.

$$
a^{3 w+1}\left|\bar{\Psi}_{0}\right| \leq \frac{1}{2(3 w+1)}
$$

that translates, in the case $w=0$ into

$$
a \bar{\Psi}_{0} \leq \frac{1}{2}
$$

We are now in condition to clarify the relation between the perturbative expansion discussed in the present Section and the general quasi-isotropic gradient expansion derived in Eqs. (4.8) and (4.12). The conclusion will be that, if the positivity of $\operatorname{Tr} K^{2}$ is enforced, the two expansions are exactly equivalent, in the sense that the expansion discussed in the present Section is just as sub-case of the general quasi-isotropic expansion.

For this purpose let us then consider Eq. (4.12) and let use the freedom to specify $\alpha_{i j}$ in Eq. (4.12) and let us choose

$$
\alpha_{i j}(\vec{x})=e^{-2 f(\vec{x})} \delta_{i j}
$$

Then, the spatial curvature tensor can be immediately computed using Eq. (5.18) and Eq. (2.3). Thus, recalling Eq. (4.6), $\mathcal{P}_{i}^{j}$ can be easily computed:

$$
\mathcal{P}_{i}^{j}=e^{2 f}\left[\nabla^{2} f \delta_{i}^{j}+\partial_{i} \partial^{j} f-(\nabla f)^{2} \delta_{i}^{j}+\partial_{i} f \partial^{j} f\right] .
$$

Consequently, from Eq. (4.8), the tensor $\mathcal{B}_{i j}$ will be

$\mathcal{B}_{i}^{j}=-\frac{4 e^{2 f}}{H_{0}^{2}(3 w+1)(3 w+5)}\left\{\left[\frac{3 w(w+1)}{(9 w+5)} \nabla^{2} f-\frac{3 w^{2}+12 w+5}{2(9 w+5)}(\nabla f)^{2}\right] \delta_{i}^{j}+\partial_{i} \partial^{j} f+\partial_{i} f \partial^{j} f\right\}$. 
This expression is still difficult to compare with the parametrization employed in the present section since $\mathcal{B}_{i}^{j}$ is not traceless. Let us then separate the traceless contribution by writing $\gamma_{i j}(\vec{x}, t)$ as

$$
\gamma_{i j}(\vec{x}, t)=a^{2}(t) e^{-2 \tilde{\Psi}}\left[\delta_{i j}+\tilde{\mu}_{i j}(\vec{x}, t)\right]
$$

where $\tilde{\mu}_{i}^{i}=0$. The traceless tensor $\tilde{\mu}_{i j}$ is essentially given by the traceless part of $\beta_{i j}=$ $a^{(3 w+1)} \mathcal{B}_{i j}$ where $\mathcal{B}_{i j}$ is given by Eq. (5.20). Therefore, we can also write the solution in terms of $\tilde{\Psi}$ :

$$
\tilde{\Psi}=f(\vec{x})-\frac{1}{2} \ln \left\{1-\frac{4}{3} \frac{e^{2 f}}{(9 w+5) H_{0}^{2}} a^{3 w+1}\left[\nabla^{2} f-\frac{1}{2}(\nabla f)^{2}\right]\right\} .
$$

In the specific case $w=0$ the previous expression becomes

$$
\tilde{\Psi}=f(\vec{x})-\frac{1}{2} \ln \left\{\left[1-\frac{4}{15 H_{0}^{2}} e^{2 f} a\left[\nabla^{2} f-\frac{1}{2}(\nabla f)^{2}\right]\right\} .\right.
$$

Let us now compare Eqs. (5.22) and (5.23) with Eq. (5.8). For for sake of simplicity let us consider the case $w=0$. Therefore, in this case, $\tilde{\Psi}$ of Eq. (5.23) can be written as

$$
\tilde{\Psi}=f(\vec{x})-\frac{1}{2} \ln \left[1-2 a \bar{\Psi}_{0}\right],
$$

where $\Psi_{0}(\vec{x})$ is the same one defined in Eq. (5.15) and it includes the contribution of the spatial gradients. Recalling now Eq. (5.17) we clearly see that the argument of the logarithm appearing in Eq. (5.24) is only compatible with $\operatorname{Tr} K^{2}$ being positive semidefinite, if $2 a \bar{\Psi}_{0} \leq$ 1. This implies that, as anticipated, $\tilde{\Psi}=\Psi$ within the accuracy of the approximation.

The condition coming from $\operatorname{Tr} K^{2} \geq 0$ is necessary for the consistency of the approximation of the present section. It is however not sufficient. This means that an even more restrictive condition on $\bar{\Psi}_{0}$ stems from Eq. (5.11). Indeed, using the parametrization (5.14) it follows, in the case $w=0$, that

$$
a\left|\bar{\Psi}_{0}\right| \leq \frac{1}{3}
$$

This inequality follows directly from Eq. (5.11) by recalling, from Eq. (5.14), that, for $w=0, \Psi=f+a \bar{\Psi}_{0}$. Thus $\ddot{\Psi}=\left(\dot{H}+H^{2}\right)\left|\bar{\Psi}_{0}\right| a$; using the last expression in Eq. (5.11) and recalling that $2 \dot{H}=-3 H^{2}$ (in the case of dusty matter) we Eq. (5.25) is readily obtained. As anticipated the condition (5.25) is more restrictive than the one derived in (5.17) always in the $w=0$ case. In fact, Eq. (5.25) implies $-1 / 3 \leq a \bar{\Psi}_{0} \leq 1 / 3$ while Eq. (5.17) implies only that $a \bar{\Psi}_{0}<1 / 2$.

The last step of our discussion is to show that not only $\tilde{\Psi}=\Psi$ but also that $\mu_{i j}=\tilde{\mu}_{i j}$. This is immediate recalling that $\tilde{\mu}_{i j}$ is constructed from the traceless part of $\beta_{i j}$ whose tensor structure in given, in terms of the intrinsic curvature tensor, by Eq. (4.8). Bearing now in mind Eq. (5.18), it is immediate to show that $\tilde{\mu}_{i j}$ equals $\mu_{i j}$ given in Eq. (5.9). 


\section{Deceleration parameter(s)}

In this section we will be concerned mainly with the calculation of the deceleration parameter. In particular we want to show that the deceleration parameter for a matter-dominated Universe can never become negative. In a complementary perspective one could say that if the deceleration parameter becomes negative, within the gradient expansion discussed in the present paper, the conditions of validity of the perturbative expansion must be violated.

Let us therefore start from the general definition of the deceleration parameter valid in the inhomogeneous case and reported in Eq. (2.10). Then let us consider the parametrization of Eq. (5.14) and in the case $w=0$. Inserting Eq. (5.14) into Eq. (2.10) we obtain

$$
q(\vec{x}, t)=-1+\frac{3 / 2-a \bar{\Psi}_{0} / 2}{\left(1-2 a \bar{\Psi}_{0}\right)}=\frac{3 a \bar{\Psi}_{0}+1}{2\left(1-2 a \bar{\Psi}_{0}\right)} .
$$

In order to derive this result, we inserted Eq. (5.14) into $K, \dot{K}$ and $\operatorname{Tr} K^{2}$ appearing in the general definition (2.10). Then we used the relation $2 \dot{H}=-3 H^{2}$ (valid in the dustdominated case). Finally, we consistently neglected the terms $\dot{\Psi}^{2}$ that, as explained in the previous Section, are of higher order since they contain 4 gradients. This means, in practice, that $\operatorname{Tr} K^{2}=H^{2}\left(1-2 a \bar{\Psi}_{0}\right)$. Concerning Eq. (6.1) the following comments are in order:

- as derived in the previous Section the validity of the perturbative expansion implies in the case $w=0$ that $a\left|\bar{\Psi}_{0}\right| \leq 1 / 3$ (see Eq. (5.25) and comments therein);

- therefore, it is clear from the expression of reported in the second equality of Eq. (2.10) that $q$ is always positive semi-definite for $-1 / 3 \leq a \bar{\Psi}_{0} \leq 1 / 3$;

- furthermore, when $a \bar{\Psi}_{0} \rightarrow-1 / 3$ (corresponding to the maximally underdense Universe allowed by the validity of the perturbative expansion), then $q \rightarrow 0$.

The last occurrence corresponds to a gradient-dominated Universe. This result was already derived in [31]. Therefore, the validity of the perturbative expansion forbids a negative deceleration parameter and, therefore, the acceleration of an Universe dominated by dusty matter (i.e. $w=0$ ).

Notice also that Eq. (5.25) implies an upper limit on $a(t)$, i.e.

$$
a \leq \frac{1}{3\left|\bar{\Psi}_{0}\right|}
$$

If sufficiently small values of $\bar{\Psi}_{0}$ are allowed, $a$ can be rather large. To support their argument, the authors of Ref. [24] took (in their figure) the smallest value of $\Psi_{0}$ to be $\bar{\Psi}_{0} \sim-1 / 4$. This simply means, according to Eq. (6.2) that $a \leq \frac{4}{3}$. In light of the discussion of the present paper the soundness of this results stems directly from the equivalence of the gradient expansions discussed in Sections 4 and 5. In fact, it is clear that the quasi-isotropic 
gradient expansion of Section 4 is suitable in the limit $a \rightarrow 0$, i.e. in the limit where it was correctly applied in the early sixties.

From the results of Section 4 and 5, moreover, it is clear that there are cases when the gradient expansion may be suitable to describe the large times behaviour. But these cases are precisely the ones for which $w<-1 / 3$. In this class of fluid models, as remarked in Section 4, the contribution of the gradients becomes progressively less important as the Universe expands. Unfortunately, the cases $w<-1 / 3$ correspond to an Universe that is not dust-dominated.

Finally, in order to complete our discussion, let us see what happens if we include terms of higher order in the gradient expansion and take the limit for $a \rightarrow \infty$. In this case we have that $\operatorname{Tr} K^{2}=(H-\dot{\Psi})^{2}$. Then using the parametrization of Eq. (5.14) into Eq. (2.10) we do find that the deceleration parameter is given by

$$
q(\vec{x}, t)=-1+\frac{3 / 2-a \bar{\Psi}_{0} / 2}{\left(1-a \bar{\Psi}_{0}\right)^{2}} .
$$

The conclusion of Ref. [24] is that if we extend the validity of Eq. (6.3) into the far future, the deviation of the deceleration parameter from $q=1 / 2$ becomes larger and larger approaching the asymptotic value $q=-1$. On the basis of the gradient expansion discussed in the present paper such a chain of arguments is arbitrary.

\section{Concluding remarks}

In the present paper the gradient expansion has been discussed for a generic perfect fluid with barotropic index $w$. It has been shown that formally different realizations of the gradient expansion are in fact equivalent if the perturbative expansion is correctly handled. The aim of the present analysis was to understand recent claims suggesting that perfect fluids with $w \geq 0$ can act as effective dark-energy candidates. The results of the present study imply that if $0 \leq w \leq 1$ the deceleration parameter is always positive semi-definite. In the present analysis we kept terms up to two gradients in the inhomogeneous expansion. At this order, the negativity of the deceleration parameter for matter sources with $0 \leq w \leq 1$ is just a signal of the breakdown of the perturbative expansion. The techniques discussed in the present paper may also be relevant in order to study a possible inhomogeneous (and anisotropic ) approach of big rips and sudden singularities. We leave this problem for future investigations.

Note added. After completing the present paper we became aware of astro-ph/0506534 by E. Kolb, S. Matarrese and A. Riotto. These authors study solutions of the gradient 
expansion discussed in Section 5 of the present paper. Their results are less general since they only consider a dust-dominated Universe. We checked that our solutions coincide with their solutions in the limit $w=0$ (our $f(\vec{x})$ becomes, in their notations, $5 / 3 \varphi(\vec{x})$ ). They also claim that the perturbative expansion they use (i.e. the one we discussed in Section 5 ) sensibly differs from the gradient expansion we discussed in Section 4 of the present paper. Our results show the opposite. 


\section{References}

[1] A. G. Riess et al. [Supernova Search Team Collaboration], Astrophys. J. 607, 665 (2004).

[2] J. L. Tonry et al. [Supernova Search Team Collaboration], Astrophys. J. 594, 1 (2003).

[3] A. G. Riess et al. [Supernova Search Team Collaboration], Astron. J. 116, 1009 (1998).

[4] S. Perlmutter et al. [Supernova Cosmology Project Collaboration], Astrophys. J. 517, 565 (1999).

[5] D. N. Spergel et al., Astrophys. J. 148, 175 (2003).

[6] R. R. Caldwell, R. Dave, and P. J. Steinhardt, Phys. Rev. Lett. 80, 1582 (1998).

[7] P. J. E. Peebles and B. Ratra, Astrophys. J. 325, L17 (1988).

[8] S. M. Carroll, Phys. Rev. Lett. 81, 3067 (1998).

[9] P. J. E. Peebles and A. Vilenkin, Phys. Rev. D 59, 063505 (1999).

[10] M. Giovannini, Class. Quant. Grav. 16, 2905 (1999).

[11] M. Giovannini, Phys. Rev. D 60, 123511 (1999).

[12] D. Babusci and M. Giovannini, Phys. Rev. D 60, 083511 (1999).

[13] T. Padmanabhan, Phys. Rept. 380, 235 (2003)

[14] P. J. E. Peebles and B. Ratra, Rev. Mod. Phys. 75, 559 (2003).

[15] R. R. Caldwell, M. Kamionkowski and N. N. Weinberg, Phys. Rev. Lett. 91, 071301 (2003).

[16] S. Nojiri, S. D. Odintsov and S. Tsujikawa, Phys. Rev. D 71, 063004 (2005).

[17] S. Nojiri and S. D. Odintsov, arXiv:hep-th/0505215.

[18] S. Rasanen, JCAP 0411, 010 (2004).

[19] J. D. Barrow, Class. Quant. Grav. 21, L79 (2004).

[20] J. D. Barrow, Class. Quant. Grav. 21, 5619 (2004).

[21] J. D. Barrow and C. G. Tsagas, Class. Quant. Grav. 22, 1563 (2005).

[22] E. M. Lifshitz and I. M. Khalatnikov, Sov. Phys. Usp. 6, 495 (1964).

[23] V. A. Belinskii, E. M. Lifshitz and I. M. Khalatnikov, Sov. Phys. Usp. 13, 745 (1971). 
[24] E. Kolb, S. Matarrese, A. Notari and A. Riotto, arXiv:hep-th/0503117.

[25] G. Geshnizjani, D. J. H. Chung and N. Afshordi, arXiv:astro-ph/0503553.

[26] E. Flanagan, arXiv:hep-th/0503202.

[27] C. M. Hirata and U. Seljak, arXiv:astro-ph/0503582.

[28] H. Alnes, M. Amarzguioui and O. Gron, arXiv:astro-ph/0506449.

[29] S. Rasanen, arXiv:astro-ph/0504005.

[30] E. Siegel and J. Fry, arXiv:astro-ph/0504421.

[31] M. Giovannini, hep-th/0505222 (CERN-PH-TH/2005-085).

[32] J. D. Barrow, Mon. Not. R. Astr. Soc. 178, 625 (1977); 179, 47 (1977).

[33] M. Giovannini, Phys. Rev. D 70, 103509 (2004); Class. Quant. Grav. 22, 363 (2005).

[34] T. J. Battefeld and R. Brandenberger, Phys. Rev. D 70, 121302 (2004).

[35] T. J. Battefeld and D. A. Easson, Phys. Rev. D 70, 103516 (2004).

[36] K. Tomita, Prog. Theor. Phys. 67, 1076 (1982).

[37] K. Tomita, Phys. Rev. D 48, 5634 (1993).

[38] N. Deruelle and D. Goldwirth, Phys. Rev. D 51, 1563 (1995).

[39] N. Deruelle and K. Tomita, Phys. Rev. D 50, 7216 (1994).

[40] G. Comer, N. Deruelle, D. Langlois, and J. Parry, Phys. Rev. D 49, 2759 (1994).

[41] E. M. Lifshitz and I. M. Khalatnikov, Sov. Phys. JETP 12, 108 (1960).

[42] E. M. Lifshitz and I. M. Khalatnikov, Sov. Phys. JETP 12, 558 (1961).

[43] V. A. Belinskii and I. M. Khalatnikov, Sov. Phys. JETP 30, 1174 (1970).

[44] I. M. Khalatnikov and E. M. Lifshitz, Phys. Rev. Lett. 24, 76 (1970).

[45] V. A. Belinskii and I. M. Khalatnikov, Sov. Phys. JETP 36, 591 (1973). 